ISSN 1420-3049

http://www.mdpi.org

\title{
Easy and Safe Preparations of (Diacetoxyiodo)arenes from Iodoarenes, with Urea-Hydrogen Peroxide Adduct (UHP) as the Oxidant and the Fully Interpreted ${ }^{1} \mathrm{H}$ - and ${ }^{13} \mathrm{C}-\mathrm{NMR}$ Spectra of the Products ${ }^{\dagger}$
}

\section{Agnieszka Zielinska and Lech Skulski *}

Chair and Laboratory of Organic Chemistry, Faculty of Pharmacy, Medical University, 1 Banacha Street, PL 02-097 Warsaw, Poland

$\dagger$ This short paper was presented at the Seventh Electronic Conference of Synthetic Organic Chemistry (ECSOC-7, http://www.mdpi.net/ecsoc-7), November 1-30, 2003 (paper A001).

* To whom correspondence should be addressed; E-mail: lechskulski@yahoo.com; Tel./Fax: (+48) 22 5720643;

Received: 30 April 2004; in revised form: 12 January 2005 / Accepted: 13 January 2005 / Published: 31 January 2005

\begin{abstract}
An easy and safe, though only moderately effective method is presented for preparing (diacetoxyiodo)arenes, $\operatorname{ArI}(\mathrm{OAc})_{2}$, from iodoarenes, ArI, using the commercially available and easily handled urea-hydrogen peroxide adduct (UHP) as the oxidant. The reactions take place in anhydrous $\mathrm{AcOH} / \mathrm{Ac}_{2} \mathrm{O} / \mathrm{AcONa}$ (a catalyst) mixtures, at $40{ }^{\circ} \mathrm{C}$ for $3.5 \mathrm{~h}$ to afford the purified $\mathrm{ArI}(\mathrm{OAc})_{2}$ in $37-78 \%$ yields. The fully interpreted ${ }^{1} \mathrm{H}$ - and ${ }^{13} \mathrm{C}-\mathrm{NMR}$ spectra of the $\mathrm{ArI}(\mathrm{OAc})_{2}$ products are reported.
\end{abstract}

Keywords: (Diacetoxyiodo)arenes, iodoarenes, urea-hydrogen peroxide adduct as oxidant, ${ }^{1} \mathrm{H}$ - and ${ }^{13} \mathrm{C}$-NMR spectra

\section{Introduction}

(Diacetoxyiodo)arenes, $\operatorname{ArI}(\mathrm{OAc})_{2}$, and particularly the parent compound (diacetoxyiodo)benzene, $\mathrm{PhI}(\mathrm{OAc})_{2}$, have been known for a long time [1,2]. They are used in organic synthesis as potent, often chemoselective, oxidizing agents. They are also used for facile syntheses of several classes of aromatic 
hypervalent iodine compounds, e.g. [bis(trifluoroacetoxy)iodo]arenes, [hydroxy(tosyloxy)iodo]arenes, aromatic iodonium salts [3], etc. In our two latest reviews [4,5] we relate and explain our previous novel syntheses of $\mathrm{ArI}(\mathrm{OAc})_{2}$ from corresponding ArI, as well our novel aromatic iodination methods, and preparations of several classes of aromatic hypervalent iodine compounds, easily attainable from aromatic iodides; particularly see ref. 4, pp. 1343-1345 and 1352-1354.

During the course of our systematic studies on effective and easy preparations of $\operatorname{ArI}(\mathrm{OAc})_{2}$ from the corresponding ArI [4, 5], we have devised several methods for their syntheses. In this work we have oxidized seven iodoarenes (Table 1) in anhydrous $\mathrm{AcOH} / \mathrm{Ac}_{2} \mathrm{O}$ mixtures; the reactions did not proceed in the absence of sodium acetate, $\mathrm{AcONa}$ (used in a stoichiometric quantity) - cf. our former work [6], where its presence in the reaction mixtures was also indispensable. The reactions took place as follows:

$$
\left.\mathrm{ArI}+[\text { urea }] \cdots \mathrm{H}_{2} \mathrm{O}_{2} \underset{3.5 \mathrm{~h} ; 40{ }^{\circ} \mathrm{C}}{\stackrel{\mathrm{AcOH} \mathrm{Ac}_{2} \mathrm{O} / \mathrm{AcONa}}{\longrightarrow}} \mathrm{ArI}(\mathrm{OAc})_{2}+\text { acetylurea }\right]+\mathrm{H}_{2} \mathrm{O}
$$

In our opinion, the novel method presented in this paper is easy and safe, albeit only moderately effective (yields are in the $37-78 \%$ range). For the first time, we have used UHP as the oxidant. This compound, which is now commercially available, may be considered a "dry carrier" of the unstable and hazardous hydrogen peroxide. The UHP solid is easy to handle, safe and stable at room temperature. Its ability to release oxidative species in water and organic media has made it a useful reagent in organic synthesis [7].

\section{Experimental}

\section{General}

Melting points submitted in the Table 1 are uncorrected. All the reagents were purchased from Aldrich or Lancaster and were used without further purification. The NMR spectra were run in $\mathrm{CDCl}_{3}$ solutions at room temperature, with TMS as an internal standard; the spectra were recorded on a Brucker AVANCE DMX 400 spectrometer. To obtain better assignments, also ${ }^{1} \mathrm{H}-{ }^{13} \mathrm{C}$ NMR correlation spectra were recorded.

Optimized Procedure for Preparing (Diacetoxyiodo)arenes from Iodoarenes:

Urea-Hydrogen Peroxide adduct, 98\% (3.02 g, $31.5 \mathrm{mmol}, 350 \%$ excess) was added portionwise to a stirred mixture of glacial $\mathrm{AcOH}(24 \mathrm{~mL})$ with $\mathrm{Ac}_{2} \mathrm{O}(9 \mathrm{~mL})$. An appropriate iodoarene $(7 \mathrm{mmol})$ was slowly added, the solution was cooled to $10-15{ }^{\circ} \mathrm{C}$, and powdered AcONa (1.26 g, $\left.15 \mathrm{mmol}\right)$ was suspended. Stirring at $40{ }^{\circ} \mathrm{C}$ was continued for $3.5 \mathrm{~h}$. After cooling, water $(35 \mathrm{~mL})$ was slowly added with stirring. The precipitated $\operatorname{ArI}(\mathrm{OAc})_{2}$ were collected by filtration, washed on the filter with a cold $\left(5-10{ }^{\circ} \mathrm{C}\right) 10 \%$ aq. $\mathrm{AcOH}$, and air-dried in the dark; if necessary, they were recrystallized from $\mathrm{AcOEt} / \mathrm{Ac}_{2} \mathrm{O}(9: 1, \mathrm{v} / \mathrm{v})$. The oily or semisolid products were extracted with $\mathrm{CH}_{2} \mathrm{Cl}_{2}$, the combined 
extracts were dried over anhydrous $\mathrm{Na}_{2} \mathrm{SO}_{4}$ and filtered, the solvent was distilled off under vacuum, and the solidified residues were recrystallized from $\mathrm{AcOEt} / \mathrm{Ac}_{2} \mathrm{O}(9: 1)$. The purities and homogeneities of the purified $\operatorname{ArI}(\mathrm{OAc})_{2}$ were firstly checked by TLC, and next confirmed by their melting points, all close to those reported in the literature (Table 1). Their chemical structures were fully supported by the ${ }^{1} \mathrm{H}-\mathrm{NMR}$ (Table 2) and ${ }^{13} \mathrm{C}-\mathrm{NMR}$ (Table 3) spectra (in $\mathrm{CDCl}_{3}$ ).

Table 1. Final yields and melting points (uncorrected) of the purified (diacetoxyiodo)arenes obtained from the corresponding iodoarenes.

\begin{tabular}{|c|c|c|c|c|}
\hline Substrate & Product & $\begin{array}{c}\text { Yield } \\
(\%)\end{array}$ & $\begin{array}{c}\text { Mp } \\
\left({ }^{\circ} \mathbf{C}\right)\end{array}$ & $\begin{array}{c}\text { Lit. } \mathbf{M p} \\
\left({ }^{\circ} \mathbf{C}\right)\end{array}$ \\
\hline $\mathrm{C}_{6} \mathrm{H}_{5} \mathrm{I}$ & $\mathrm{C}_{6} \mathrm{H}_{5} \mathrm{I}(\mathrm{OAc})_{2}$ & 44 & $161-162$ & $161-163[6]$ \\
$3-\mathrm{FC}_{6} \mathrm{H}_{4} \mathrm{I}$ & $3-\mathrm{FC}_{6} \mathrm{H}_{4} \mathrm{I}(\mathrm{OAc})_{2}$ & 37 & $143-145$ & $144-145[8]$ \\
$4-\mathrm{FC}_{6} \mathrm{H}_{4} \mathrm{I}$ & $4-\mathrm{FC}_{6} \mathrm{H}_{4} \mathrm{I}(\mathrm{OAc})_{2}$ & 78 & $177-180$ & $179[8]$ \\
$2-\mathrm{MeC}_{6} \mathrm{H}_{4} \mathrm{I}$ & $2-\mathrm{MeC}_{6} \mathrm{H}_{4} \mathrm{I}(\mathrm{OAc})_{2}$ & 64 & $142-147$ & $140-142[6]$ \\
$2,4-\mathrm{Me}_{2} \mathrm{C}_{6} \mathrm{H}_{3} \mathrm{I}$ & $2,4-\mathrm{Me}_{2} \mathrm{C}_{6} \mathrm{H}_{3} \mathrm{I}(\mathrm{OAc})_{2}$ & 69 & $126-128$ & $128[9]$ \\
$2-\mathrm{MeOC}_{6} \mathrm{H}_{4} \mathrm{I}$ & $2-\mathrm{MeOC}_{6} \mathrm{H}_{4} \mathrm{I}(\mathrm{OAc})_{2}$ & 48 & $145-147$ & $147-149[6]$ \\
$3-\mathrm{MeOC}_{6} \mathrm{H}_{4} \mathrm{I}$ & $3-\mathrm{MeOC}_{6} \mathrm{H}_{4} \mathrm{I}(\mathrm{OAc})_{2}$ & 57 & $130-132$ & $133-135[6]$ \\
\hline
\end{tabular}

Table 2. ${ }^{1} \mathrm{H}-\mathrm{NMR}$ chemical shifts for pure (diacetoxyiodo)arenes. Cf. our present experimental values with those previously reported [11].

\begin{tabular}{|c|c|c|c|c|c|c|c|c|c|}
\hline \multirow{2}{*}{ Product } & \multicolumn{9}{|c|}{ Chemical shifts $\delta$ (ppm) } \\
\hline & $\mathbf{H}_{2}$ & $\mathbf{H}_{3}$ & $\mathbf{H}_{4}$ & $\mathbf{H}_{5}$ & $\mathbf{H}_{6}$ & $\mathrm{OCH}_{3}$ & $\begin{array}{c}\mathrm{CH}_{3} \\
\text { (o) } \\
\end{array}$ & $\begin{array}{c}\mathrm{CH}_{3} \\
(p) \\
\end{array}$ & $\begin{array}{r}\mathrm{CH}_{3} \\
\text { in OAc } \\
\end{array}$ \\
\hline $\mathrm{C}_{6} \mathrm{H}_{5} \mathrm{I}(\mathrm{OAc})_{2}$ & $\begin{array}{c}8.10 \\
\mathrm{~d}\end{array}$ & $\begin{array}{c}7.50 \\
\mathrm{t}\end{array}$ & $\begin{array}{c}7.60 \\
t\end{array}$ & $\begin{array}{c}7.50 \\
t\end{array}$ & $\begin{array}{c}8.10 \\
\mathrm{~d}\end{array}$ & - & - & - & $\begin{array}{c}2.01 \\
\mathrm{~S}\end{array}$ \\
\hline $3-\mathrm{FC}_{6} \mathrm{H}_{4} \mathrm{I}(\mathrm{OAc})_{2}$ & $\begin{array}{c}7.29 \\
\mathrm{~d} \\
\end{array}$ & - & $\begin{array}{c}7.84 \\
\mathrm{t} \\
\end{array}$ & $\begin{array}{c}7.51 \\
\mathrm{~m} \\
\end{array}$ & $\begin{array}{c}7.84 \\
\mathrm{t} \\
\end{array}$ & - & - & - & $\begin{array}{c}2.02 \\
\mathrm{~s} \\
\end{array}$ \\
\hline $4-\mathrm{FC}_{6} \mathrm{H}_{4} \mathrm{I}(\mathrm{OAc})_{2}$ & $\begin{array}{c}8.09 \\
\mathrm{~d} \\
\end{array}$ & $\begin{array}{c}7.19 \\
\mathrm{t} \\
\end{array}$ & - & $\begin{array}{c}7.19 \\
\mathrm{t} \\
\end{array}$ & $\begin{array}{c}8.09 \\
\mathrm{~d} \\
\end{array}$ & - & - & - & $\begin{array}{c}2.02 \\
\mathrm{~s} \\
\end{array}$ \\
\hline $2-\mathrm{MeC}_{6} \mathrm{H}_{4} \mathrm{I}(\mathrm{OAc})_{2}$ & - & $\begin{array}{c}7.52 \\
\mathrm{~d} \\
\end{array}$ & $\begin{array}{c}7.52 \\
\mathrm{t} \\
\end{array}$ & $\begin{array}{c}7.26 \\
\mathrm{t} \\
\end{array}$ & $\begin{array}{c}8.17 \\
\mathrm{~d} \\
\end{array}$ & - & $\begin{array}{c}2.72 \\
\mathrm{~s} \\
\end{array}$ & - & $\begin{array}{c}1.98 \\
\mathrm{~s}\end{array}$ \\
\hline $2,4-\mathrm{Me}_{2} \mathrm{C}_{6} \mathrm{H}_{3} \mathrm{I}(\mathrm{OAc})_{2}$ & - & $\begin{array}{c}7.31 \\
\mathrm{~s} \\
\end{array}$ & - & $\begin{array}{c}7.05 \\
\mathrm{~d} \\
\end{array}$ & $\begin{array}{c}8.04 \\
\mathrm{~d} \\
\end{array}$ & - & $\begin{array}{c}2.68 \\
\mathrm{~s} \\
\end{array}$ & $\begin{array}{c}2.40 \\
\mathrm{~s} \\
\end{array}$ & $\begin{array}{c}1.98 \\
\mathrm{~s} \\
\end{array}$ \\
\hline $2-\mathrm{MeOC}_{6} \mathrm{H}_{4} \mathrm{I}(\mathrm{OAc})_{2}$ & - & $\begin{array}{c}7.16 \\
\mathrm{~d} \\
\end{array}$ & $\begin{array}{c}7.59 \\
\mathrm{t} \\
\end{array}$ & $\begin{array}{c}7.04 \\
\mathrm{t} \\
\end{array}$ & $\begin{array}{c}8.14 \\
\mathrm{~d} \\
\end{array}$ & $\begin{array}{c}3.99 \\
\mathrm{~s} \\
\end{array}$ & - & - & $\begin{array}{c}1.97 \\
\mathrm{~s} \\
\end{array}$ \\
\hline $3-\mathrm{MeOC}_{6} \mathrm{H}_{4} \mathrm{I}(\mathrm{OAc})_{2}$ & $\begin{array}{c}7.65 \\
\mathrm{~s}\end{array}$ & - & $\begin{array}{c}7.10 \\
\mathrm{~d}\end{array}$ & $\begin{array}{c}7.41 \\
\mathrm{t}\end{array}$ & $\begin{array}{c}7.67 \\
\mathrm{~s}\end{array}$ & $\begin{array}{c}3.87 \\
\text { S }\end{array}$ & - & - & $\begin{array}{c}2.02 \\
\mathrm{~s}\end{array}$ \\
\hline
\end{tabular}


Table 3. ${ }^{13} \mathrm{C}$ NMR chemical shifts for pure (diacetoxyiodo)arenes and coupling constants $J^{19} \mathrm{~F}-^{13} \mathrm{C}$ and $J^{19} \mathrm{~F}-\mathrm{C}-{ }^{13} \mathrm{C}(\mathrm{Hz})$. Cf. our present experimental values with those previously reported incompletely $[10,12]$.

\begin{tabular}{|c|c|c|c|c|c|c|}
\hline \multirow{2}{*}{ Product } & \multicolumn{6}{|c|}{ Chemical shifts of aromatic $\mathrm{C}$ atoms in $\delta(\mathbf{p p m})$} \\
\hline & $\mathbf{C}_{1}$ & $\mathbf{C}_{2}$ & $\mathbf{C}_{3}$ & $\mathrm{C}_{4}$ & $\mathbf{C}_{5}$ & $\mathrm{C}_{6}$ \\
\hline \multirow{2}{*}{$\mathrm{C}_{6} \mathrm{H}_{5} \mathrm{I}(\mathrm{OAc})_{2}$} & 121.58 & 134.94 & 130.96 & 131.74 & 130.96 & 134.94 \\
\hline & $\mathrm{s}$ & $\mathrm{s}$ & $\mathrm{s}$ & $\mathrm{s}$ & $\mathrm{s}$ & $\mathrm{s}$ \\
\hline \multirow[b]{2}{*}{$3-\mathrm{FC}_{6} \mathrm{H}_{4} \mathrm{I}(\mathrm{OAc})_{2}$} & 120.65 & 122.56 & 161.33 & 119.26 & 132.33 & 130.87 \\
\hline & $\mathrm{s}$ & $\begin{array}{c}\mathrm{d} \\
J=24.6\end{array}$ & $\begin{array}{c}\mathrm{d} \\
J=253.0\end{array}$ & $\begin{array}{c}\mathrm{d} \\
J=20.7\end{array}$ & $\mathrm{~s}$ & $\mathrm{~S}$ \\
\hline \multirow[b]{2}{*}{$4-\mathrm{FC}_{6} \mathrm{H}_{4} \mathrm{I}(\mathrm{OAc})_{2}$} & 115.64 & 137.74 & 118.59 & 164.63 & 118.82 & 135.95 \\
\hline & $\mathrm{s}$ & $\mathrm{s}$ & $\begin{array}{c}\mathrm{d} \\
J=22.6\end{array}$ & $\begin{array}{c}\mathrm{d} \\
J=253.7\end{array}$ & $\begin{array}{c}\mathrm{d} \\
J=22.6\end{array}$ & s \\
\hline \multirow{2}{*}{$2-\mathrm{MeC}_{6} \mathrm{H}_{4} \mathrm{I}(\mathrm{OAc})_{2}$} & 127.29 & 140.72 & 128.51 & 130.97 & 132.82 & 137.30 \\
\hline & $\mathrm{s}$ & $\mathrm{s}$ & $\mathrm{s}$ & $\mathrm{s}$ & $\mathrm{s}$ & $\mathrm{s}$ \\
\hline \multirow{2}{*}{$2,4-\mathrm{Me}_{2} \mathrm{C}_{6} \mathrm{H}_{3} \mathrm{I}(\mathrm{OAc})_{2}$} & 124.09 & 143.73 & 131.78 & 140.66 & 129.40 & 137.32 \\
\hline & $\mathrm{s}$ & $\mathrm{s}$ & $\mathrm{s}$ & $\mathrm{s}$ & $\mathrm{s}$ & $\mathrm{s}$ \\
\hline \multirow{2}{*}{$2-\mathrm{MeOC}_{6} \mathrm{H}_{4} \mathrm{I}(\mathrm{OAc})_{2}$} & 113.63 & 156.46 & 112.33 & 134.70 & 122.91 & 137.94 \\
\hline & $\mathrm{s}$ & $\mathrm{s}$ & $\mathrm{s}$ & $\mathrm{s}$ & $\mathrm{s}$ & $\mathrm{s}$ \\
\hline \multirow{2}{*}{$3-\mathrm{MeOC}_{6} \mathrm{H}_{4} \mathrm{I}(\mathrm{OAc})_{2}$} & 121.62 & 120.68 & 160.72 & 118.14 & 131.73 & 127.25 \\
\hline & $\mathrm{s}$ & $\mathrm{s}$ & $\mathrm{s}$ & $\mathrm{s}$ & $\mathrm{s}$ & $\mathrm{s}$ \\
\hline
\end{tabular}

\begin{tabular}{|c|c|c|c|c|c|}
\hline \multirow[b]{2}{*}{ Product } & \multicolumn{5}{|c|}{ Chemical shifts of $C$ atoms in substituents in $\delta(\mathrm{ppm})$} \\
\hline & $\begin{array}{c}\mathrm{CH}_{3} \\
(\boldsymbol{o})\end{array}$ & $\begin{array}{c}\mathrm{CH}_{3} \\
(p)\end{array}$ & $\mathrm{OCH}_{3}$ & $\begin{array}{c}\mathrm{CH}_{3} \\
\text { in } \mathrm{OAc}\end{array}$ & $\begin{array}{c}\mathrm{C}=\mathrm{O} \\
\text { in OAc }\end{array}$ \\
\hline $\mathrm{C}_{6} \mathrm{H}_{5} \mathrm{I}(\mathrm{OAc})_{2}$ & - & - & - & $\begin{array}{c}20.36 \\
\text { s }\end{array}$ & $\begin{array}{c}176.39 \\
\mathrm{~s}\end{array}$ \\
\hline $3-\mathrm{FC}_{6} \mathrm{H}_{4} \mathrm{I}(\mathrm{OAc})_{2}$ & - & - & - & $\begin{array}{c}20.51 \\
\mathrm{~S}\end{array}$ & $\begin{array}{c}176.76 \\
\mathrm{~s}\end{array}$ \\
\hline $4-\mathrm{FC}_{6} \mathrm{H}_{4} \mathrm{I}(\mathrm{OAc})_{2}$ & - & - & - & $\begin{array}{c}20.47 \\
\mathrm{~s}\end{array}$ & $\begin{array}{c}176.66 \\
\mathrm{~s}\end{array}$ \\
\hline $2-\mathrm{MeC}_{6} \mathrm{H}_{4} \mathrm{I}(\mathrm{OAc})_{2}$ & $\begin{array}{c}25.68 \\
\mathrm{~s}\end{array}$ & - & - & $\begin{array}{c}20.42 \\
\mathrm{~s}\end{array}$ & $\begin{array}{c}176.53 \\
\mathrm{~s}\end{array}$ \\
\hline $2,4-\mathrm{Me}_{2} \mathrm{C}_{6} \mathrm{H}_{3} \mathrm{I}(\mathrm{OAc})_{2}$ & $\begin{array}{c}25.56 \\
\mathrm{~s}\end{array}$ & $\begin{array}{c}21.64 \\
\mathrm{~s}\end{array}$ & - & $\begin{array}{c}20.52 \\
\mathrm{~s}\end{array}$ & $\begin{array}{c}176.57 \\
\mathrm{~s}\end{array}$ \\
\hline $2-\mathrm{MeOC}_{6} \mathrm{H}_{4} \mathrm{I}(\mathrm{OAc})_{2}$ & - & - & $\begin{array}{c}57.06 \\
\mathrm{~s}\end{array}$ & $\begin{array}{c}20.54 \\
\text { S }\end{array}$ & $\begin{array}{c}176.82 \\
\mathrm{~s}\end{array}$ \\
\hline $3-\mathrm{MeOC}_{6} \mathrm{H}_{4} \mathrm{I}(\mathrm{OAc})_{2}$ & - & - & $\begin{array}{c}55.92 \\
\mathrm{~s}\end{array}$ & $\begin{array}{c}20.56 \\
\mathrm{~s}\end{array}$ & $\begin{array}{c}176.64 \\
\mathrm{~s}\end{array}$ \\
\hline
\end{tabular}




\section{References}

1. Willgerodt, C. Zur Kenntnis aromatischer Jodidchloride, des Jodoso- und Jodobenzols. Ber. Dtsch. Chem. Ges. 1892, 25, 3494-3502.

2. Willgerodt, C. Die organischen Verbindungen mit mehrwertigem Jod; Enke: Stuttgart, 1914.

3. Varvoglis, A. The Organic Chemistry of Polycoordinated Iodine; VCH: Weinheim, 1992.

4. Skulski, L. Organic Iodine(I, III, V) Chemistry: 10 Years of Development at the Medical University of Warsaw, Poland (1990-2000). Molecules 2000, 5, 1331-1371; Avail. at URL: http://www.mdpi.org/molecules/papers/51201331.pdf

5. Skulski, L. Novel Easy Preparations of Some Aromatic Iodine(I, III, V) Reagents, Widely Applied in Modern Organic Synthesis. Molecules 2003, 8, 45-52; Avail. at URL: http://www.mdpi.org/molecules/papers/80100045.pdf

6. Skulski, L. ; Kazmierczak, P. ; Kraszkiewicz, L. Syntheses of (Diacetoxyiodo)arenes or Iodylarenes from Iodoarenes, with Sodium Periodate as the Oxidant. Molecules 2001, 6, 881-891; Avail. at URL: http://www.mdpi.org/molecules/papers/61200881.pdf

7. a) Lancaster Research Chemicals 2004-2005, p. 1605; see references for the applications of UHP in organic synthesis; b) Heaney, H. Oxidation Reactions Using Magnesium Monoperphthalate and Urea Hydrogen Peroxide. Aldrichim. Acta 1993, 26, 35-45; c) Heaney, H. Novel Organic Peroxygen Reagents for Use in Organic Synthesis. Top. Curr. Chem. 1993, 164 (Organic Peroxygen Chemistry), 1-19.

8. Lyalin, V. V. ; Syrova, G. P. ; Orda, V. V. ; Alekseeva, L. A. ; Yagupol'skii, L. M. Electronic Nature of Iodine-containing Substituents (in Russian). Zh. Org. Khim. 1970, 6, 1420-1422; J. Org. Chem. USSR (Engl. Transl.) 1970, 6, 1433-1435.

9. Beringer, F. M. ; Gindler, E. M. Organic Compounds of Polyvalent Iodine. Iodine Abstr. and Revs. 1956, 3, No. 3, 1-70.

10. Katritzky, A. R. ; Gallos, J. K. ; Durst, H. D. Structure of and Electronic Interactions in Aromatic Polyvalent Iodine Compounds: a Carbon-13 NMR Study. Magn. Reson. Chem. 1989, 27, 815822.

11. Kazmierczak, P.; Skulski, L. A Simple, Two-step Conversion of Various Iodoarenes to (Diacetoxyiodo)arenes with Chromium(VI) Oxide as the Oxidant. Synthesis (Stuttgart) 1998, 1721-1723.

12. Cerioni, G. ; Uccheddu, G. Solution Structure of Bis(acetoxy)iodoarenes as Observed by ${ }^{17} \mathrm{O}$ NMR Spectroscopy. Tetrahedron Lett. 2004, 45, 505-507.

Sample Availability: The samples of pure (diacetoxyiodo)arenes, prepared one year ago and kept in a fridge, have deteriorated in full or in part. Hence, they are not available.

(C) 2005 by MDPI (http:www.mdpi.org). Reproduction is permitted for noncommercial purposes. 